\title{
Gauge theories on the random-block lattice
}

\author{
T.W. Chiu \\ Physics Department, California Institute of Technology, Pasadena, CA 91125, USA \\ and Physics Department, National Taiwan University, Taipei, Taiwan 10764, ROC
}

Received 22 April 1990

\begin{abstract}
Gauge theories are formulated on the random-block lattice in which the sites are cartesian product of $D$ sets of random coordinates, one from each dimension, where $D$ is the dimension of the space-time. Some numerical results of the pure gauge theories are presented.
\end{abstract}

Two years ago, the properties of the free Dirac fermion fields have been studied on the four-dimensional random-block lattice ( $R B L)$ [1]. It has been shown that the RBL may provide a natural way to resolve the fermion doubling problem. Recently, the fermion propagator and the vacuum polarization tensor of the Schwinger model have been studied on the RBL [2], in the free field limit and the weak coupling perturbation theory, respectively. These results support the possibility of having a chiral fermion interacting with the dynamical gauge field, on the RBL, with the correct continuum limit. It is natural to extend the investigations to incorporating dynamical gauge fields. In this paper, the gauge theories are formulated on the RBL, and Monte Carlo simulations are performed for $\mathrm{U}(1), \mathrm{SU}(2)$ and $\mathrm{SU}(3)$ pure gauge theories respectively. The average energy per plaquette and the specific heat are measured respectively.

The sites of a 4D RBL are the cartesian product of four sets of random coordinates, one from each dimension. On a $4 \mathrm{D}$ RBL, the link variable pointing from the site $i$ to the site $i+\mu$ is

$U_{\mu}(i)=\exp \left[\mathrm{i} g l_{i}^{\mu} A_{\mu}\left(x_{i}+\frac{1}{2} l_{i}^{\mu}\right)\right], \quad l_{i}^{\mu}=x_{i+\mu}-x_{i}$,

and the action of the $\mathrm{SU}(N)$ pure gauge theory is

$A=\beta \sum_{\mathrm{p}} w_{\mathrm{p}}\left(1-\frac{1}{N} \operatorname{Re} \operatorname{tr} U_{\mathrm{p}}\right) \equiv \beta E$,

where $U_{\mathrm{p}}$ is the ordered product of the link variables around the plaquette $\mathrm{p}, \beta=2 \mathrm{~N} / \mathrm{g}^{2}$ and $w_{\mathrm{p}}$ is the weight of the plaquette $\mathrm{p}$, which is the ratio of the area dual to $\mathrm{p}$ and the area of $\mathrm{p}$,

$w_{\mathrm{p}}=\tilde{A}_{\mathrm{p}} / A_{\mathrm{p}}, \quad A_{\mathrm{p}}=l_{i}^{\mu} l_{i}^{\nu}, \quad \tilde{A}_{\mathrm{p}} A_{\mathrm{p}}=l_{i}^{(1)} l_{i}^{(2)} l_{i}^{(3)} l_{i}^{(4)}$.

In the continuum limit, this action goes to the continuum gauge field action

$A_{\mathrm{C}}=\int \mathrm{d}^{4} x_{4}^{\frac{1}{4}} F_{\mu \nu}^{a} F_{\mu \nu}^{a}$.

If $O(U)$ is a function of the link variables $U$, then its quantum expectation value is

$\langle O\rangle=\frac{1}{V^{N_{\mathrm{s}}}} \int \prod_{i=1}^{N_{\mathrm{s}}} \mathrm{d}^{4} x_{i} \frac{\int \prod_{l=1}^{N_{t}} \mathrm{~d} U_{l} O(\{x\},\{U\}) \exp [-A(\{x\},\{U\})]}{\int \prod_{l=1}^{N_{f}} \mathrm{~d} U_{l} \exp [-A(\{x\},\{U\})]}$,

This research was partially supported by US Department of Energy and by National Science Council, ROC. 
where $N_{\mathrm{s}}$ is the number of sites, $N_{\ell}=4 N_{\mathrm{s}}$ is the number of links, $N_{\mathrm{p}}=6 N_{\mathrm{s}}$ is the number of plaquettes and $V$ is the volume of the lattice. The observables calculated in this paper are the average energy per plaquette, $e(\beta)$ and its derivative, specific heat $C_{\mathrm{v}}=-\beta^{2} \mathrm{de} / \mathrm{d} \beta$.

In the strong coupling limit $\beta \rightarrow 0$, expansion in terms of $\beta$ yields

$$
e(\beta)=\frac{E(\beta)}{N_{\mathrm{p}}}=\left\langle w_{\mathrm{p}}\right\rangle-\frac{\beta}{2 N^{2}} b_{N}\left\langle w_{\mathrm{p}}^{2}\right\rangle+\mathrm{O}\left(\beta^{2}\right)
$$

where

$$
b_{N}=1+\delta_{N, 2}, \quad\left\langle w_{\mathrm{p}}\right\rangle=\frac{1}{V^{N_{\mathrm{s}}}} \int \prod_{i=1}^{N_{s}} \mathrm{~d}^{4} x_{i} \frac{1}{N_{\mathrm{p}}} \sum_{\mathrm{p}} w_{\mathrm{p}}, \quad\left\langle w_{\mathrm{p}}^{2}\right\rangle=\frac{1}{V^{N_{s}}} \int \prod_{i=1}^{N_{s}} \mathrm{~d}^{4} x_{i} \frac{1}{N_{\mathrm{p}}} \sum_{\mathrm{p}} w_{\mathrm{p}}^{2} .
$$

In the weak coupling limit, $\beta \rightarrow \infty$, we can expand the action around $U_{\mathrm{p}}=1$. To fix the gauge, we select a maximally connected tree and then set all links on it to be one. Then the number of link variables appearing in the action is $N_{\ell}-N_{\mathrm{s}}+1=3 N_{\mathrm{s}}+1 \simeq 3 N_{\mathrm{s}}$. According to the equipartition theorem, each degree of freedom contributes $1 / 2 \beta$ to the total energy. Thus we have the average energy per plaquette

$e(\beta)=n_{\mathrm{G}} / 4 \beta$,

where $n_{\mathrm{G}}$ is the number of generators of the gauge group $\mathrm{G}$.

Monte Carlo simulations are performed to calculate $e(\beta)$ and $C_{v}$ for a range of values of $\beta$. The Metropolis algorithm [3], the Creutz heat bath [4] and the Cabibbo-Marinari heat bath [5] are used for the U(1), SU(2) and SU(3) pure gauge theories respectively. A small RBL of $4^{4}$ sites has been employed for simulations. The separation between two neighboring sites in any direction is greater than $0.5 a$ and less than $1.5 a$, where $a$ is the average lattice spacing. This condition is imposed to avoid the occurrence of a huge plaquette weight which would spoil the statistics of the Monte Carlo simulations. The plaquette weights are normalized such that their sum is equal to the number of plaquettes. The results of average energy per plaquette are plotted in figs. 1a, $1 \mathrm{~b}$ and $1 \mathrm{c}$ for $\mathrm{U}(1), \mathrm{SU}(2)$ and $\mathrm{SU}(3)$ theories, respectively. At each $\beta, 1100$ sweeps are performed and the initial 100 sweeps are used for thermalization. The heating process is denoted by circles while the cooling process is denoted by crosses. These results are almost the same as their counterparts on a regular lattice, except the crossovers from strong to weak coupling regimes in $\mathrm{SU}(2)$ and $\mathrm{SU}(3)$ are more smooth on a random-block lattice than on a regular lattice. Fig. 1a shows that the $U(1)$ theory has a first order phase transition around $\beta=1.2$. The specific heat is plotted in figs. $2 \mathrm{a}, 2 \mathrm{~b}$ and $2 \mathrm{c}$ respectively, and has a peak in all cases, in contrast to Ren's [6] results on a random lattice where the specific heat curve for $\mathrm{SU}(2)$ has no peak.

The action of QED on a 4D RBL can be written as

$$
A_{\mathrm{L}}=\sum_{i} \sum_{\mu} \omega_{i} K_{i}^{\mu}\left[\bar{\psi}_{i} \gamma_{\mu} U_{\mu}(i) \psi_{i+\mu}-\bar{\psi}_{i} \gamma_{\mu} U_{\mu}^{+}(i-\mu) \psi_{i-\mu}\right]+m \sum_{i} \omega_{i} \bar{\psi}_{i} \psi_{i}+\beta \sum_{\mathrm{p}} w_{\mathrm{p}}\left[1-\operatorname{Re}\left(U_{\mathrm{p}}\right)\right]
$$

where $\psi_{i}$ and $\bar{\psi}_{i}$ are two independent four-component spinors at site $i, \omega_{i}$ is the weight at site $i, K_{i}^{\mu}$ is the inverse of the distance between the sites $i+\mu$ and $i-\mu$, and other symbols were defined above. In terms of the coordinates of sites, $\omega_{i}$ and $K_{i}^{\mu}$ can be written as

$\omega_{i}=\frac{1}{16}\left(x_{i_{1}+1}-x_{i_{1}-1}\right)\left(x_{i_{2}+1}-x_{i_{2}-1}\right)\left(x_{i_{3}+1}-x_{i_{3}-1}\right)\left(x_{i_{4}+1}-x_{i_{4}-1}\right), \quad K_{i}^{\mu}=\left(x_{i+\mu}-x_{i-\mu}\right)^{-1}$.

In the continuum limit (8) goes to the continuum QED action

$\boldsymbol{A}_{\mathrm{c}}=\int \mathrm{d}^{4} x\left[\bar{\psi}(x) \gamma_{\mu}\left(\partial_{\mu}+\mathrm{i} g \boldsymbol{A}_{\mu}+m\right) \psi(x)+\frac{1}{4} F_{\mu \nu} F_{\mu \nu}\right]$.

The $n$-point Green's function of fermion fields is

$$
G_{n}\left(x_{1}, \ldots, x_{n}\right)=\frac{1}{V^{N_{s}-n}} \int \prod_{i=1}^{N_{j}} \mathrm{~d}^{4} y_{i} \prod_{j=1}^{n} \delta^{4}\left(y_{j}-x_{j}\right) G_{n}^{\mathrm{L}}\left(y_{1}, \ldots, y_{n}\right) \text {, }
$$


U(1) Pure Gauge Theory on a $4^{4}$ random-block lattice SU(2) Pure Gauge Theory on a $4^{4}$ random-block lattice
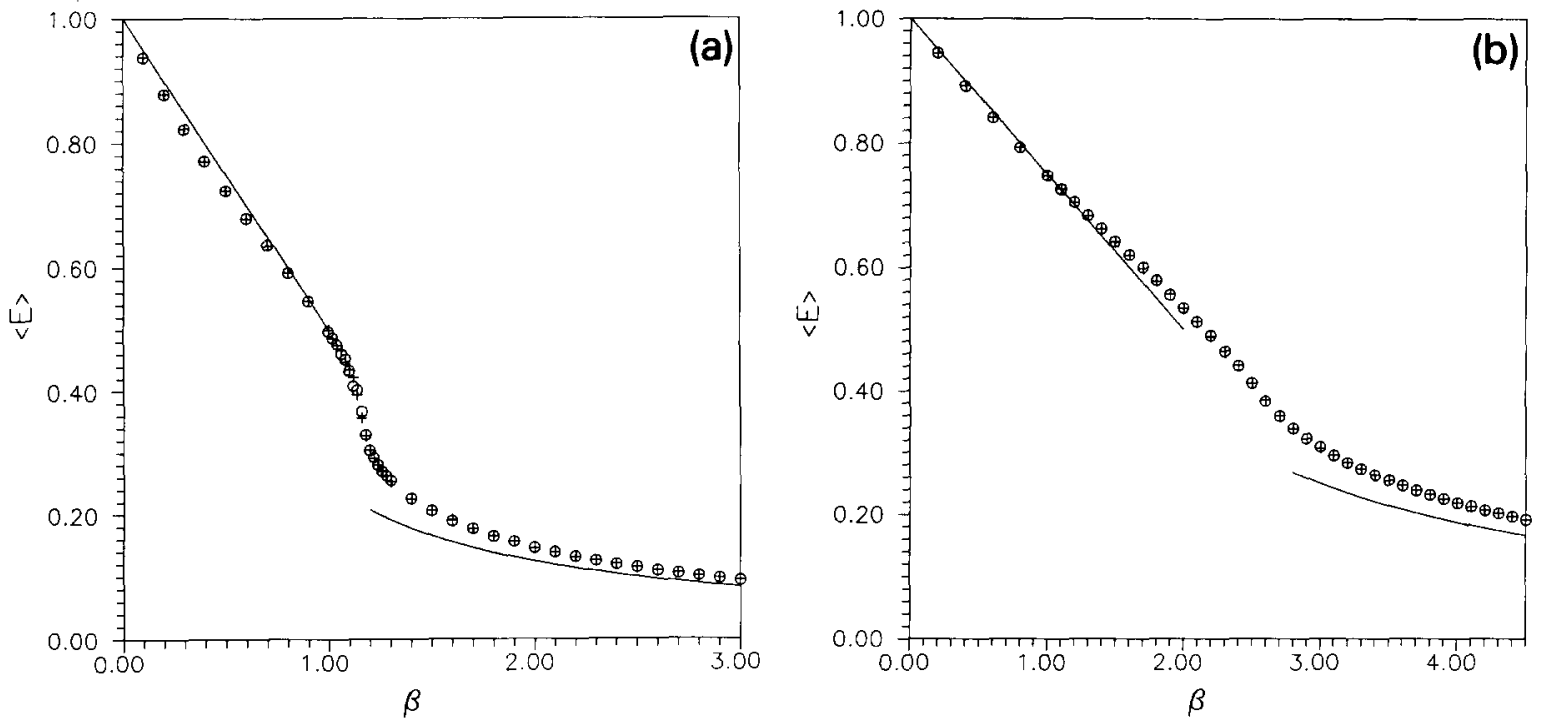

SU(3) Pure Gauge Theory on a $4^{4}$ random-block lattice

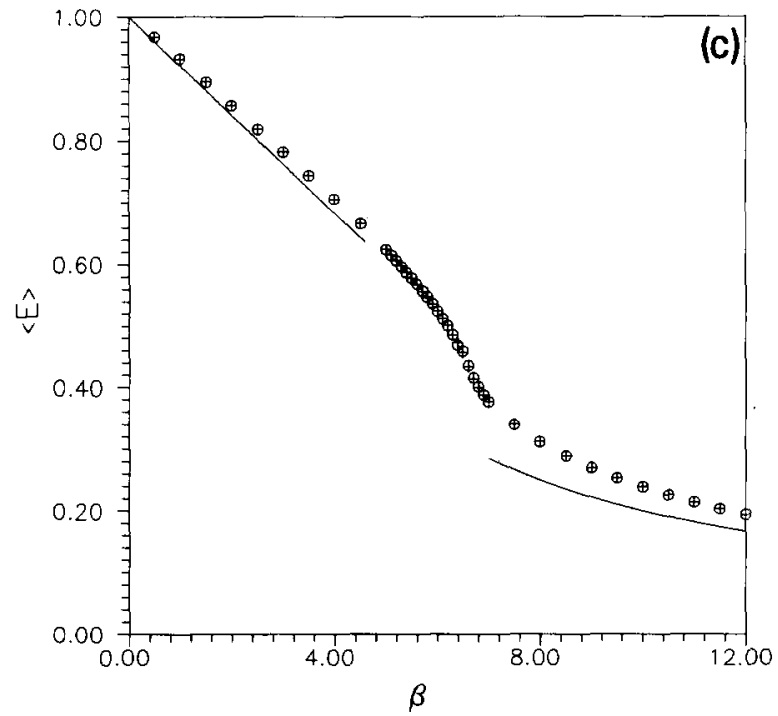

Fig. 1. The average energy per plaquette of pure gauge theories on a $4^{4} \mathrm{RBL}$. The solid lines are the strong coupling expansion and the weak coupling expansion given by $(6 \mathrm{a})$ and (7) respectively. The heating process is denoted by circles while the cooling process by crosses. (a) $U(1)$. (b) $S U(2)$. (c) $S U(3)$.

where

$G_{n}^{\mathrm{L}}\left(y_{1}, \ldots, y_{n}\right)=\frac{\int \prod_{i} \mathrm{~d} \psi_{i} \mathrm{~d} \bar{\psi}_{i} \prod_{i, \mu} \mathrm{d} U_{\mu}(i) \exp \left(-A_{\mathrm{L}}\right) \bar{\psi}_{1} \cdots \bar{\psi}_{k} \cdots \psi_{l} \cdots \psi_{n}}{\int \prod_{i} \mathrm{~d} \psi_{i} \mathrm{~d} \bar{\psi}_{i} \prod_{i, \mu} \mathrm{d} U_{\mu}(i) \exp \left(-A_{\mathrm{L}}\right)}$,

and $V=L^{4}$ is the volume of the lattice. 

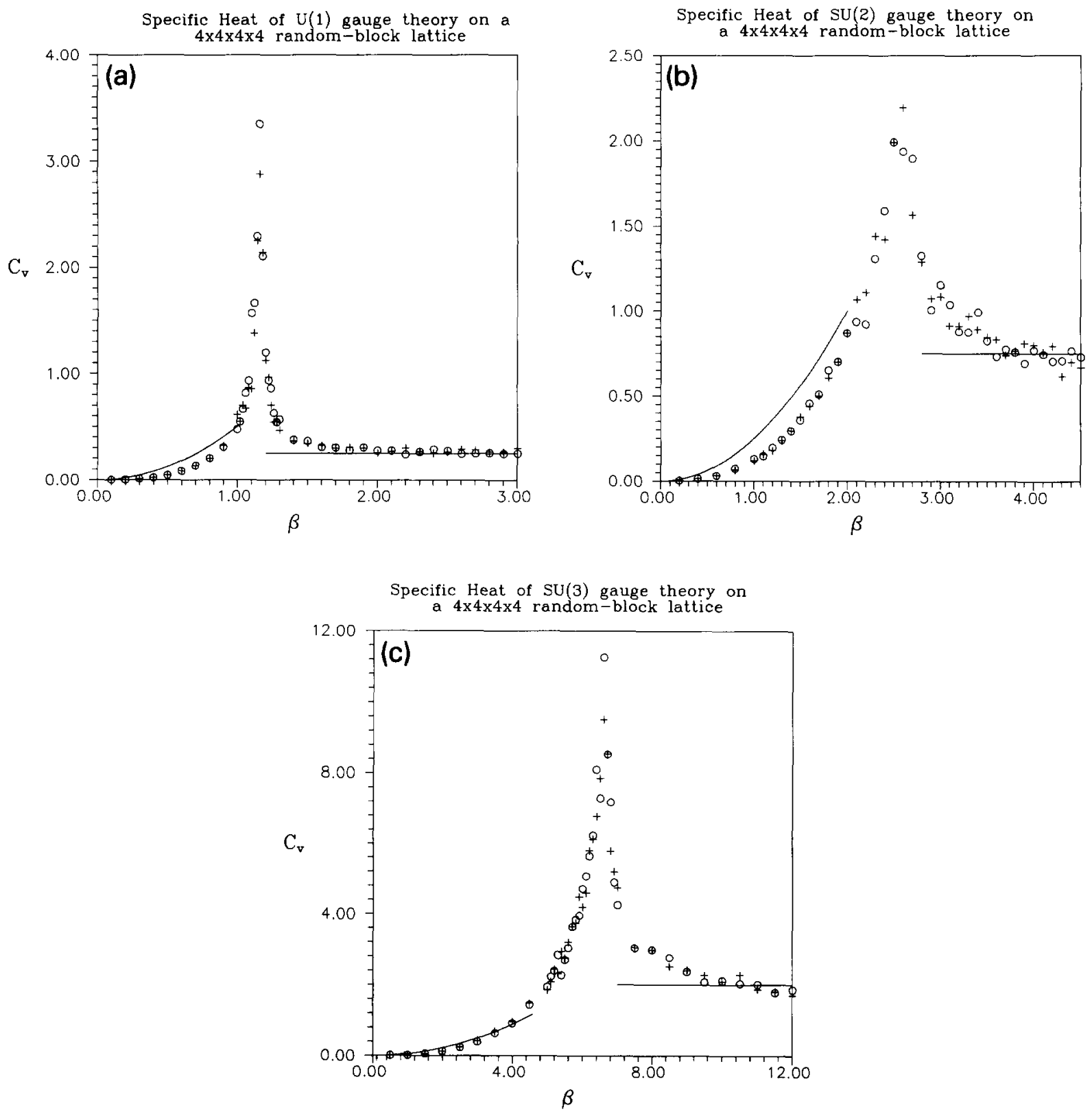

Fig. 2. The specific heat of pure gauge theories on a $4^{4} \mathrm{RBL}$. The solid lines are the strong coupling expansion and the weak coupling expansion respectively. The heating process is denoted by circles while the cooling process by crosses. (a) U(1). (b) SU(2). (c) SU(3).

The action of the abelian Higgs model on a 4D RBL can be written as

$$
\begin{aligned}
A_{\mathrm{L}} & =\sum_{i} \sum_{\mu} 2 \omega_{i}\left(l_{i}^{\mu} l_{i-\mu}^{\mu}\right)^{-1} \phi_{i}^{*} \phi_{i}+\sum_{i} \omega_{i}\left[m^{2} \phi_{i}^{*} \phi_{i}+\lambda\left(\phi_{i}^{*} \phi_{i}\right)^{2}\right] \\
& -\sum_{i} \sum_{\mu} \lambda_{i \mu}\left[\phi_{i}^{*} U_{\mu}(i) \phi_{i+\mu}+\text { h.c. }\right]+\beta \sum_{\mathrm{p}} w_{\mathrm{p}}\left[1-\operatorname{Re}\left(U_{\mathrm{p}}\right)\right],
\end{aligned}
$$


where $\phi_{i}$ and $\phi_{i}^{*}$ are two independent complex scalar fields at site $i, \lambda_{i \mu}$ is the link weight of the link $(i, i+\mu)$ $\lambda_{i \mu}=\frac{1}{8}\left(l_{i}^{\mu}\right)^{-1} \prod_{\nu \neq \mu}\left(x_{i+\nu}-x_{i-\nu}\right)$,

and other symbols were defined above. In the continuum limit the lattice action goes to the familiar abelian-Higgs model

$A_{\mathrm{c}}=\int \mathrm{d}^{4} x\left[\left(\mathrm{D}_{\mu} \phi\right)^{*} \mathrm{D}^{\mu} \phi+m^{2} \phi^{*} \phi+\lambda\left(\phi^{*} \phi\right)^{2}+\frac{1}{4} F_{\mu \nu} F^{\mu \nu}\right]$.

It is simple to generalize the action of QED or the abelian Higgs model to other non-abelian gauge models. Monte Carlo simulations of QED and the abelian Higgs model are now in progress.

I am grateful to Professor Geoffrey C. Fox for his support and very kind hospitality during my stay at CALTECH.

\section{References}

[1] T.W. Chiu, Phys. Lett. B 206 (1988) 510.

[2] T.W. Chiu, Phys. Lett. B 217 (1989) 151.

[3] N. Metropolis, A.W. Rosenbluth, M. N. Rosenbluth, A.H. Teller and E. Teller, J. Chem. Phys. 21 (1953) 1087.

[4] M. Creutz, Phys. Rev. D 21 (1980) 2308.

[5] N. Cabibbo and E. Marinari, Ptys. Lett. B 119 (1982) 387.

[6] H.C. Ren, Nucl. Phys. B 235 (1984) 321. 